\title{
SIMPLE NEAR-RINGS ASSOCIATED WITH MEROMORPHIC PRODUCTS
}

\author{
C. J. MAXSON AND K. C. SMITH
}

(Communicated by Donald S. Passman)

\begin{abstract}
Let $H$ be a subgroup of $G^{2}$ and let
$M_{0}(G, 2, H)=\left\{f \in M_{0}(G) \mid f(H) \subseteq H\right\}$.

In this paper we characterize in terms of properties of $H$ when $M_{0}(G, 2, H)$ is a simple near-ring.
\end{abstract}

\section{INTRODUCTION}

Let $G$ be a group written additively and $k$ a positive integer, $k \geq 2$. R. Remak has pointed out in [5] that one can construct subgroups of the direct power $G^{k}$ as follows. For $j \in\{1,2, \ldots, k\}$, let $B_{j}$ be a subgroup of $G, \bar{B}_{j}$ a normal subgroup of $B_{j}$ such that $B_{j} / \bar{B}_{j} \cong B_{j+1} / \bar{B}_{j+1}$ with isomorphisms $\sigma_{j}$, $j \in\{1, \ldots k-1\}$. Let $\alpha$ be an ordinal, $\left\{b_{1 \eta} \mid \eta<\alpha\right\}$ a set of coset representatives of $\bar{B}_{1}$ in $B_{1}$ where $b_{10}=0$ and define a subset $H \subseteq G^{k}$ by $H=\bigcup_{\eta<\alpha}\left[\left(b_{1 \eta}+\bar{B}_{1}\right) \times \prod_{j=1}^{k-1}\left(\sigma_{j} \circ \sigma_{j-1} \circ \cdots \circ \sigma_{1}\left(b_{1 \eta}+\bar{B}_{1}\right)\right)\right]$. Here $H$ is called a $k$-fold meromorphic product and will be denoted by $H=B_{1} / \bar{B}_{1}{\stackrel{\times}{\sigma_{1}}}_{B_{2}} / \bar{B}_{2}{\stackrel{\times}{\sigma_{2}}}$ $\ldots \stackrel{\times}{\sim}_{\sigma_{k-1}} B_{k} / \bar{B}_{k}$. It is straightforward to verify that $H$ is a subgroup of $G^{k}$. However, only for $k=2$ can every subgroup of $G^{k}$ be obtained as a meromorphic product.

Theorem I.1 (Klein-Fricke) [5]. Every subgroup of $G \times G$ is a 2-fold meromorphic product.

Let $M(G)=\{f: G \rightarrow G\}$ act on $G^{k}$ componentwise, i.e. let $f\left(x_{1}, x_{2}, \ldots\right.$, $\left.x_{k}\right)=\left(f\left(x_{1}\right), \ldots, f\left(x_{k}\right)\right)$. For any subgroup $H$ of $G^{k}$ we define $M(G, k, H)$ $=\{f \in M(G) \mid f(H) \subseteq H\}$. In a similar manner, let $M_{0}(G)=\{f: G \rightarrow$ $G \mid f(0)=0\}$ and define $M_{0}(G, k, H)=\left\{f \in M_{0}(G) \mid f(H) \subseteq H\right\}$. These $M(G, k, H)$ and $M_{0}(G, k, H)$ are subnear-rings of $M(G)$ with identity id: $G \rightarrow G, \operatorname{id}(x)=x, \forall x \in G$.

Received by the editors October 17, 1988.

1980 Mathematics Subject Classification (1985 Revision). Primary 16A76; Secondary 20 E07. 
H. Wielandt [6], suggested relating the properties of $M(G, k, H)$ and $M_{0}(G, k, H)$ with those of the subgroup $H$. Because of the above theorem much more can be said for the case $k=2$ and henceforth we restrict ourselves to this case.

Recall, for any group $G$, with $|G|>2, M(G)$ and $M_{0}(G)$ are simple nearrings. (For this and other basic information about near-rings the reader is referred to the book by Meldrum [3] or the book by Pilz [4].) As one might expect, the situation for $M(G, 2, H)$ and $M_{0}(G, 2, H)$ is much different from that of $M(G)$ and $M_{0}(G)$. In fact, as we shall see, neither of these near-rings need be simple. Further when $M_{0}(G, 2, H)$ is simple, it need not be the case that $M(G, 2, H)$ is simple.

Example 1.2. Let $G=\mathbb{Z}_{2}+\mathbb{Z}_{2}, H=G / A \stackrel{\times}{\sim}_{\sigma} G / B$ where $A=\mathbb{Z}_{2}+\{0\}$ and $B=\{0\}+\mathbb{Z}_{2}$. By calculations, or using Theorem III.12 we see that $M_{0}(G, 2, H)$ is simple. However, if $c_{a}: G \rightarrow G$ denotes the constant function $c_{a}(x)=a$, $\forall x \in G$ then $M_{c}(G, 2, H)=\left\{c_{(0,0)}, c_{(1,1)}\right\}$ is an ideal of $M(G, 2, H)$. Thus $M_{0}(G, 2, H)$ is simple but $M(G, 2, H)$ is not.

It is the purpose of this paper to characterize in terms of properties of the subgroup $H$ when $M_{0}(G, 2, H)$ is simple. In the next section we present some general results which reduce the problem to a special case. In the final section we focus on this particular case.

\section{General Results}

Let $H=B_{1} / \bar{B}_{1} \stackrel{{ }_{\sim}}{\sim} B_{2} / \bar{B}_{2}$. We first show that in some special situations, $M_{0}(G, 2, H)$ and $M(G, 2, H)$ are always simple. In fact if $\bar{B}_{1} \cap \bar{B}_{2}=$ $G$ then $H=G / G \stackrel{\searrow}{\sim}_{\sigma} G / G$ so $H=G \times G$ and $M_{0}(G, 2, H)=M_{0}(G)$ while $M(G, 2, H)=M(G)$. Thus $M_{0}(G, 2, H)$ is simple and $M(G, 2, H)$ is simple when $|G|>2$. If $B_{1} \cup B_{2}=\{0\}$ then $H=\{(0,0)\}$ so $M_{0}(G, 2, H)=$ $M(G, 2, H)=M_{0}(G)$. Thus we take $\bar{B}_{1} \cap \bar{B}_{2} \neq G$ and $B_{1} \cup B_{2} \neq\{0\}$. We now give an easy but basic result used throughout the paper.

Lemma II.1. Let $H=B_{1} / \bar{B}_{1} \stackrel{\times}{\sim}_{\sigma} B_{2} / \bar{B}_{2}$ and let $f \in M_{0}(G, 2, H)$. Then $f\left(\bar{B}_{i}\right) \subseteq \bar{B}_{i}, i=1,2$.

Proof. For $\bar{b}_{1} \in \bar{B}_{1},\left(\bar{b}_{1}, 0\right) \in H$. Therefore $\left(f\left(\bar{b}_{1}\right), 0\right) \in H$ which implies $f\left(\bar{b}_{1}\right) \in \bar{B}_{1}$.

Another useful tool is given next. The proof is omitted since the result is well known.

Lemma II.2. Let $N_{0}=M_{0}(G, 2, H)$ and let $U$ be a subset of $G$ with the property $f(U) \subseteq U, \forall f \in N_{0}$. Define $\operatorname{Ann}_{N_{0}}(U)=\left\{f \in N_{0} \mid f(U)=\{0\}\right\}$. Then $\operatorname{Ann}_{N_{0}}(U)$ is an ideal of $N_{0}$.

Lemma II.3. If $B_{1} \cup B_{2} \neq G$ then $M_{0}(G, 2, H)$ is not simple. 
Proof. Let $U=B_{1} \cup B_{2}$ and note that $f(U) \subseteq U, \forall f \in M_{0}(G, 2, H)$ and so by the above lemma, $I=\operatorname{Ann}_{N_{0}}(U)$ is an ideal in $M_{0}(G, 2, H)$. Moreover, since id $\notin I, I \varsubsetneqq M_{0}(G, 2, H)$. Further, $I \neq\{0\}$ since the function $h: G \rightarrow G$ given by $h(x)=0$ if $x \in U$ and $h(x)=x$ if $x \notin U$ is in $I$.

We have $B_{1} \cup B_{2} \neq\{0\}$. Suppose now one of $B_{1}, B_{2}$ is $\{0\}$, say $B_{1}=\{0\}$, so $H=\{0\} /\{0\} \stackrel{\times}{\sim} B_{2} / B_{2}$. Thus $M_{0}(G, 2, H)=M(G, 2, H)$. If $M_{0}(G, 2, H)$ is simple, then from the above lemma, $B_{2}=G$, hence $H=\{0\} \times G$ and $M(G, 2, H)=M_{0}(G, 2, H)=M_{0}(G)$. Conversely if $B_{2}=G, M_{0}(G, 2, H)=$ $M_{0}(G)$ is simple.

Corollary II.4. Let $H=\{0\} /\{0\} \stackrel{\times}{{ }_{\sigma}} B_{2} / B_{2}$. Then $M(G, 2, H)=M_{0}(G, 2, H)$ and $M_{0}(G, 2, H)$ is simple $\Leftrightarrow B_{2}=G$.

Henceforth we also take $B_{1} \neq\{0\} \neq B_{2}$. In the following result we collect some necessary conditions for $M_{0}(G, 2, H)$ to be simple.

Proposition II.5. Let $H=B_{1} / \bar{B}_{1} \underset{\sigma}{\stackrel{\times}{\sim}} B_{2} / \bar{B}_{2}$ and let $N_{0}=M_{0}(G, 2, H)$ be simple. Then

(i) $\bar{B}_{1} \cap \bar{B}_{2}=\{0\}$;

(ii) if $B_{1} \neq G, H=B_{1} / \bar{B}_{1} \stackrel{\times}{\sigma}_{\sigma} G /\{0\}$;

(iii) if $G$ is finite, $H=G / \bar{B}_{1} \stackrel{\times}{\sigma}_{\sigma} G / \bar{B}_{2}$.

Proof. (i) We take $U=\bar{B}_{1} \cap \bar{B}_{2}$ and suppose $U \neq\{0\}$. From Lemma II.1, $f(U) \subseteq U$ and so $I=\operatorname{Ann}_{N_{0}}(U)$ is an ideal, $I \neq N_{0}$. Let $\bar{b}$ be a nonzero element in $U$ and define $h: G \rightarrow G$ by $h(x)=0, x \in U, h(x)=\bar{b}, x \notin U$. Then $h \neq 0$ and $h \in I$, a contradiction to the simplicity of $N_{0}$.

(ii) From Lemma II.3, $B_{1} \cup B_{2}=G$ and since $B_{1} \neq G$ we must have $B_{2}=G$. Assume $\bar{B}_{2} \neq\{0\}$ and choose $0 \neq \bar{b}_{2} \in \bar{B}_{2}$. Define $h: G \rightarrow G$ by $h(x)=0$, $x \in B_{1}$, and $h(x)=\bar{b}_{2}$ if $x \notin B_{1}$. For $(u, v) \in H$,

$$
(f(u), f(v))= \begin{cases}(0,0), & v \in B_{1}, \\ \left(0, \bar{b}_{2}\right), & v \notin B_{1} .\end{cases}
$$

Thus $h \in N_{0}$ and for $U=B_{1},\{0\} \varsubsetneqq \operatorname{Ann}_{N_{0}}(U) \varsubsetneqq N_{0}$, a contradiction.

(iii) From Lemma II.3, $B_{1}=G$ or $B_{2}=G$, say $B_{1}=G$. If $B_{2} \neq G$ then from (ii), $H=G /\{0\} \stackrel{\times}{\sim}_{\sigma} B_{2} / \bar{B}_{2}$ which is impossible when $G$ is finite. Thus we must have $B_{2}=G$ and so $H=G / \bar{B}_{1} \stackrel{\times}{\sim}_{\sigma} G / \bar{B}_{2}$.

Convention. For the remainder of this paper we take $G$ to be a finite group, written additively with identity 0 . We let $S^{*}=S \backslash\{0\}$ for any subset $S$ of $G$. We also let $N_{0}$ denote $M_{0}(G, 2, H)$.

From (iii) of the above proposition we need only consider subgroups $H$ of the form $H=G / A \stackrel{\times}{\sim}_{\sigma} G / B$ where $A \cap B=\{0\}$. We handle first the case $A=\{0\}$. 
Theorem II.6. Let $H=G / A \stackrel{\searrow}{\sim}_{\sigma} G / B, A=\{0\}$. Then $B=\{0\}$ and considering $\sigma$ as an automorphism of $G$ we have $N_{0}=\left\{f \in M_{0}(G) \mid f \sigma=\sigma f\right\}$. Moreover, $N_{0}$ is simple $\Leftrightarrow$ all $\mathscr{A}$-orbits of $G^{k}$ have the same size where $\mathscr{A}=\langle\sigma\rangle$, the cyclic group of automorphisms of $G$ generated by $\sigma$.

Proof. Since $G$ is finite, $A=\{0\}$ implies $B=\{0\}$ so $H=\{(a, \sigma a) \mid a \in G\}$ where we consider $\sigma$ as an automorphism of $G$. But $f \in N_{0} \Leftrightarrow(f(a), f \sigma(a)) \in$ $H$. However $(f(a), u) \in H \Leftrightarrow u=\sigma f(a)$, so $N_{0}=\left\{f \in M_{0}(G) \mid f \sigma=\sigma f\right\}$, the centralizer near-ring $\mathscr{C}(\mathscr{A} ; G)$ where $\mathscr{A}=\langle\sigma\rangle$. The final statement now follows from Theorem 1.1 of [2].

We conclude this section with a general result about functions in $M(G, 2, H)$ and obtain as a corollary a result to be used in the remaining case.

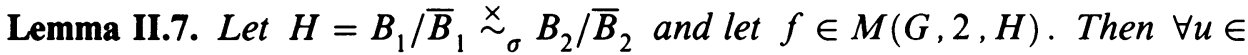
$B_{1}, f\left(u+\bar{B}_{1}\right) \subseteq f(u)+\bar{B}_{1}$ and $\forall v \in B_{2}, f\left(v+\bar{B}_{2}\right) \subseteq f(v)+\bar{B}_{2}$.

Proof. Let $\sigma\left(u+\bar{B}_{1}\right)=x+\bar{B}_{2}$ and $\sigma\left(f(u)+\bar{B}_{1}\right)=y+\bar{B}_{2}$. Since $(u, x) \in H$, $(f(u), f(x)) \in H$ so $f(x) \in y+\bar{B}_{2}$. But then for every $b \in \bar{B}_{1},(u+b, x) \in H$ implies $(f(u+b), f(x)) \in H$, and since $f(x) \in y+\bar{B}_{2}, f(u+b) \in f(u)+\bar{B}_{1}$. Similarly we get $f\left(v+\bar{B}_{2}\right) \subseteq f(v)+\bar{B}_{2}$.

Corollary II.8. Let $H=G / A \stackrel{\times}{\sim}_{\sigma} G / B$ with $A \cap B=\{0\}$. If $f \in N_{0}, a \in A$, $b \in B, f(a+b)=f(a)+f(b)$.

Proof. Since $A$ and $B$ are normal subgroups of $G$ with $A \cap B=\{0\}, a+b=$ $b+a$. From the above lemma, $f(a+b)=f(a)+\bar{b}, \bar{b} \in B$ and $f(b+a)=$ $f(b)+\bar{a}, \bar{a} \in A$. Since $a+b=b+a, f(a)+\bar{b}=f(b)+\bar{a}=\bar{a}+f(b)$ and since $A \cap B=\{0\}, \bar{a}=f(a), \bar{b}=f(b)$.

\section{THE CASE $H=G / A \stackrel{\times}{\sim}_{\sigma} G / B, A \neq\{0\}$}

We start this section by defining two ascending chains of normal subgroups of $G$ which play an important role in determining whether or not $N_{0}$ is simple. Let $A_{0}=A$. Let $A_{1}$ be the subgroup of $G$ such that $A_{1} / A=\sigma^{-1}((A+B) / B)$. Then $A_{1}$ is a normal subgroup of $G$ and $A_{0} \subseteq A_{1}$. Inductively we define $A_{j}$ to be the subgroup of $G$ such that $A_{j} / A=\sigma^{-1}\left(\left(A_{j-1}+B\right) / B\right)$. These $A_{j}$ are normal subgroups of $G$ and $A_{j-1} \subseteq A_{j}$. Analogously we define $B_{0}=B, B_{1}$ to be the subgroup of $G$ such that $\sigma((B+A) / A)=B_{1} / B$ and again inductively $B_{j}$ to be the subgroup of $G$ such that $\sigma\left(\left(B_{j-1}+A\right) / A\right)=B_{j} / B$. The $B_{j}$ are normal subgroups of $G$ with $B_{j-1} \subseteq B_{j}$. Since $G$ is finite these ascending chains must eventually become stationary.

Let $H=G / A \stackrel{\times}{\sim}_{\sigma} G / B, A \neq\{0\}$. Since $G$ is finite, $|A|=|B|$ and also $\exists$ integer $l \geq 0$ such that $A_{l}=A_{l+1}=\cdots=A_{l+t}$ for each integer $t, t \geq$ 0 . From $\sigma^{-1}\left(\left(A_{j}+B\right) / B\right)_{=} A_{j+1} / A$ and the fact that $|A|=|B|$ we obtain 
$\left|A_{j}+B\right|=\left|A_{j+1}\right|$ and by symmetry $\left|B_{j}+A\right|=\left|B_{j+1}\right|$. Since $\left|A_{l}\right|=\left|A_{l+1}\right|=\mid A_{l}+$ $B|=| A_{l}|| B|/| A_{l} \cap B \mid$ we get $\left|A_{l} \cap B\right|=|B|$, so $B_{0}=B \subseteq A_{l}$. Suppose $B_{k} \subseteq A_{l}$. By definition, $\sigma^{-1}\left(B_{k+1} / B\right)=\left(B_{k}+A\right) / A \subseteq A_{l} / A=A_{l+1} / A=\sigma^{-1}\left(\left(A_{l}+B\right) / B\right)$. Hence $B_{k+1} / B \subseteq\left(A_{l}+B\right) / B$ which implies $B_{k+1} \subseteq A_{l}$. Thus, if for some $m$, $B_{m}=G$ then for some $n, A_{n}=G$. Since the above argument is symmetric in $A$ and $B$ we have the following.

Lemma III.1. $\exists n, A_{n}=G \Leftrightarrow \exists m, B_{m}=G$.

If there exists an integer $n$ such that $A_{n}=G$ then there is a least such integer $n$. In this case we say $G$ has A-length $n$ and denote this by $n=A l(G)$. In a symmetric manner we define the $B$-length of $G$. The previous lemma says that $G$ has an $A$-length $\Leftrightarrow G$ has a $B$-length.

We show now that the subgroups $A_{i}$ and $B_{j}$ are $N_{0}$-invariant. By Lemma II.7 $A_{0}=A$ and $B_{0}=B$ are $N_{0}$-invariant. If $a_{1} \in A_{1}$ then there exists an $a \in A_{0}$ such that $\sigma\left(a_{1}+A\right)=a+B$, so $\left(a_{1}, a\right) \in H$. If $f \in N_{0}$ then $f\left(a_{1}, a\right)=$ $\left(f\left(a_{1}\right), f(a)\right) \in H$ and since $f(a) \in A_{0}$ then $f\left(a_{1}\right) \in A_{1}$. Continuing we have $f\left(A_{i}\right) \subseteq A_{i}$ by induction. Similarly for the $B_{j}$ 's.

Suppose $G$ does not have an $A$-length and let $l$ be the least non-negative integer such that $A_{l}=A_{l+1}=\cdots$ and $A_{l} \neq G$. Define $f: G \rightarrow G$ by $f(x)=0$, $x \in A_{l}$ and $f(x)=x, x \notin A_{l}$. We show $f \in N_{0}$. Let $(u, v) \in H$. If $u \in A_{l}$ then $\exists w \in A_{l-1}, b \in B$ such that $v=w+b$. But then $v \in A_{l-1}+B \subseteq A_{l}$ since we showed above $A_{l}=A_{l+1}$ implies $B \subseteq A_{l}$. Thus $(f(u), f(v))=$ $(0,0) \in H$. If $u \notin A_{l}$ then $v \notin A_{l}$, hence $(f(u), f(v))=(u, v) \in H$. Thus $f \in \operatorname{Ann}_{N_{0}}(U)$ where $U=A_{l}$ and $\{0\} \varsubsetneqq N_{0}$. This establishes the next result.

Lemma III.2. If $N_{0}$ is simple, $\exists n$ such that $n=A l(G)$.

Lemma III.3. $A \cap B=A_{1} \cap B=\cdots=A_{l} \cap B=\{0\} \Leftrightarrow A \cap B=A \cap B_{1}=\cdots=$ $A \cap B_{l}=\{0\}$.

Proof. Suppose $A \cap B=A_{1} \cap B=\cdots=A_{l} \cap B=\{0\}$. We show $A \cap B_{j}=\{0\}$, $0 \leq j \leq l$. To this end suppose $\exists x_{j} \in A \cap B_{j}, x_{j} \neq 0$. Then $\exists x_{j-1} \neq 0$ in $A_{1} \cap B_{j-1}$ such that $\left(x_{j-1}, x_{j}\right) \in H$. For if $x_{j-1}=0$ then $x_{j} \in A \cap B=\{0\}$, a contradiction. Continuing one obtains $x_{1} \neq 0$ in $A_{j-1} \cap B_{1}$. But then $\exists x_{0} \neq 0$, $x_{0} \in A_{j} \cap B$, a contradiction. The converse follows in the same manner.

Lemma III.4. Let $A \cap B=A_{1} \cap B=\cdots=A_{k-1} \cap B=\{0\}$ and $A_{k} \cap B \neq\{0\}$. Then $\forall j, 1 \leq j \leq k, A_{j} \cap B_{k-j} \neq\{0\}$ and $\exists$ isomorphisms $\sigma_{j}: A_{j} \cap B_{k-j} \rightarrow$ $A_{j-1} \cap B_{k-(j-1)}$.

Proof. As in the above lemma, $A_{k} \cap B \neq\{0\}$ implies $A_{j} \cap B_{k-j} \neq\{0\} \quad 1 \leq j \leq$ $k$. Now let $0 \neq x \in A_{j} \cap B_{k-j}, j \geq 1$. Then $\exists y \neq 0$ in $A_{j-1} \cap B_{k-(j-1)}$ such that $(x, y) \in H$. For if $y=0$, then $x \in A \cap B_{k-j}=\{0\}$, a contradiction to the above lemma. Also $y$ is unique since $(x, y),(x, \bar{y}) \in H$ implies $y-\bar{y} \in B$ and since $y-\bar{y} \in A_{j-1}$ we get $y=\bar{y}$. Thus we have a function $\sigma_{j}: A_{j} \cap$ $B_{k-j} \rightarrow A_{j-1} \cap B_{k-(j-1)}$ defined by $\sigma_{j}(x)=y$. Moreover $\sigma_{j}$ is one-one since 
$(x, y),(\bar{x}, y)$ in $H$ implies $x-\bar{x} \in A \cap B_{k-j}$. Since $j \geq 1$ this gives $x=\bar{x}$. Also, $\sigma_{j}$ is onto for if $y \in A_{j-1} \cap B_{k-(j-1)}$ then $\exists x \in A_{j} \cap B_{k-j}$ such that $(x, y) \in H$. But this means $\sigma_{j}(x)=y$. To show $\sigma_{j}$ is an isomorphism let $x_{1}, x_{2} \in A_{j} \cap B_{k-j}$. Then there exist unique $y_{1}, y_{2} \in A_{j-1} \cap B_{k-(j-1)}$ such that $\left(x_{1}, y_{1}\right),\left(x_{2}, y_{2}\right) \in H$. Since $H, A_{j} \cap B_{k-j}$, and $A_{j-1} \cap B_{k-(j-1)}$ are groups we have $\left(x_{1}+x_{2}, y_{1}+y_{2}\right) \in H$ with $\sigma_{j}\left(x_{1}+x_{2}\right)=y_{1}+y_{2}=\sigma_{j}\left(x_{1}\right)+\sigma_{j}\left(x_{2}\right)$.

Theorem III.5. If $N_{0}$ is simple then $\exists n, n=A l(G)$ and $\forall k, 0 \leq k<n$, $A_{k} \cap B=\{0\}$.

Proof. From Lemma III.2, $\exists n, n=A l(G)$. Suppose $\exists k<n$ such that $A \cap B=$ $\cdots=A_{k-1} \cap B=\{0\}$ but $A_{k} \cap B \neq\{0\}$. We show there exists a nonzero function $f \in N_{0}$ such that $f(A)=\{0\}$. But then for $U=A$ in Lemma II.2, $N_{0}$ is not simple, contrary to hypothesis.

To construct the desired function, we use the above lemma to obtain nonzero $\bar{b}_{j}$ in $A_{k-j} \cap B_{j}, 0 \leq j \leq k$ such that

$$
\left(\bar{b}_{0}, \bar{b}_{1}\right),\left(\bar{b}_{1}, \bar{b}_{2}\right), \ldots,\left(\bar{b}_{k-1}, \bar{b}_{k}\right) \in H .
$$

Let $F$ denote the $(k+1)$-tuple $\left(\bar{b}_{0}, \bar{b}_{1}, \ldots, \bar{b}_{k-1}, \bar{b}_{k}\right)$. Since $n=A l(G)$, $\exists m, m=B l(G)$ and since $A_{k-1} \cap B=\{0\}$ implies $A \cap B_{k-1}=\{0\}$ we have $m \geq k$. Let $x \in G^{*}$. Thus, there is a unique $j, 0 \leq j \leq m$ such that $x \in B_{j} \backslash B_{j-1}$ where we take $B_{-1}=\{0\}$. Let $x=b_{j}$. Then there exist elements $b_{j-1} \in B_{j-1} \backslash B_{j-2}, \ldots, b_{j-k} \in B_{j-k} \backslash B_{j-(k+1)}$ such that

$$
(++) \quad\left(b_{j-k}, b_{j-k+1}\right), \ldots,\left(b_{j-1}, b_{j}\right) \in H \text {. }
$$

We make the convention that if any of the subscripts $i$ in $(++)$ are negative then $B_{i}=\{0\}$. In the $(k+1)$-tuple, $\left(b_{j-k}, b_{j-k+1}, \ldots, b_{j}\right)$, each element $b_{i}$ is in $A_{k}$ or is not. In this way we get a $(k+1)$-tuples of 0 's and 1 's associated to $\left(b_{j-k}, \ldots, b_{j}\right)$, i.e. define $\varepsilon_{j-i}$ by

$$
\varepsilon_{j-i}= \begin{cases}0 & \text { if } b_{j-i} \in A_{k} . \\ 1 & \text { if } b_{j-i} \notin A_{k} .\end{cases}
$$

We call $\left(\varepsilon_{j-k}, \ldots, \varepsilon_{j}\right)$ the signature of $x=b_{j}$, denoted by $E(x)$. We must show $E(x)$ is well defined. To this end let $\left(b_{j-k}^{\prime}, \ldots, b_{j}^{\prime}\right)$ be another $(k+1)$ tuple associated with $x=b_{j}$, that is

$$
\left(b_{j-k}^{\prime}, b_{j-k+1}^{\prime}\right), \ldots,\left(b_{j-1}^{\prime}, b_{j}\right) \in H \quad \text { where } b_{j-i}^{\prime} \in B_{j-i} \backslash B_{j-i-1} .
$$

Note that $b_{j-1}^{\prime}-b_{j-1} \in A_{0}$ so $b_{j-1}^{\prime}=b_{j-1}+a$ and $b_{j-1}^{\prime} \in A_{k} \Leftrightarrow b_{j-1} \in A_{k}$. Then from $\left(b_{j-2}^{\prime}, b_{j-1}+a\right),\left(b_{j-2}, b_{j-1}\right) \in H$ we get $\left(b_{j-2}^{\prime}-b_{j-2}, a\right) \in H$ so $b_{j-2}^{\prime}, b_{j-2} \in A_{1}$, thus $b_{j-2}^{\prime} \in A_{k} \Leftrightarrow b_{j-2} \in A_{k}$. In general, $b_{j-i}^{\prime}-b_{j-i} \in A_{i-1}$, $1 \leq i \leq k$, so $b_{j-i}^{\prime} \in A_{k} \Leftrightarrow b_{j-i} \in A_{k}$. This shows that $E(x)$ is well defined.

We define $f: G \rightarrow G$ as follows. For $x \in G, x \in B_{j} \backslash B_{j-1}$, say $x=b_{j}$, define $f(x)=\varepsilon_{j-k} \bar{b}_{k}+\varepsilon_{j-(k-1)} \bar{b}_{k-1}+\cdots+\varepsilon_{j} \bar{b}_{0}=E(x) \cdot F$, where $E(x) \cdot F$ 
denotes the scalar product of the $(k+1)$-tuples $E(x)$ and $F$. From the previous paragraph $f$ is well defined.

Claim 1. $f\left(b_{j}+a_{0}\right)=f\left(b_{j}\right) \quad \forall a_{0} \in A$.

Since $a_{0} \in A$, for $i=1,2, \ldots, k \exists a_{i} \in A_{i} \backslash A_{i-1}$ such that $\left(a_{k}, a_{k-1}\right), \ldots$, $\left(a_{1}, a_{0}\right)$ are in $H$. Using $(++)$ we obtain

$$
\left(b_{j-k}+a_{k}, b_{j-k+1}+a_{k-1}\right), \ldots,\left(b_{j-1}+a_{1}, b_{j}+a_{0}\right) \in H .
$$

Since $a_{0}, a_{1}, \ldots, a_{k} \in A_{k}$ we have $b_{j-i}+a_{i} \in A_{k} \Leftrightarrow b_{j-i} \in A_{k}$, i.e. $E\left(b_{j}\right)=$ $E\left(b_{j}+a_{0}\right)$. Hence $f\left(b_{j}+a_{0}\right)=f\left(b_{j}\right)$.

Claim 2. $f \in N_{0}$.

Every element of $H$ has the form $\left(b_{j-1}+a, b_{j}\right)$ for some $j$ where $b_{j} \in$ $B_{j} \backslash B_{j-1}, b_{j-1} \in B_{j-1} \backslash B_{j-2}$ and $a \in A$. From Claim 1, $f\left(b_{j-1}+a\right)=f\left(b_{j-1}\right)$ so to show $f \in N_{0}$ it suffices to show $\left(f\left(b_{j-1}\right), f\left(b_{j}\right)\right) \in H$. Let $F_{1}=$ $\left(b_{j-k-1}, b_{j-k}, \ldots, b_{j-1}\right)$ be a $(k+1)$-tuple determined by $b_{j-1}$ as in $(++)$. But then $F_{2}=\left(b_{j-k}, \ldots, b_{j-1}, b_{j}\right)$ is a $(k+1)$-tuple determined by $b_{j}$ so $f\left(b_{j}\right)=E\left(b_{j}\right) \cdot F_{1}$ and $f\left(b_{j-1}\right)=E\left(b_{j-1}\right) \cdot F_{2}$. From $(+),\left(\varepsilon_{j-k} \bar{b}_{k-1}, \varepsilon_{j-k} \bar{b}_{k}\right)$, $\ldots,\left(\varepsilon_{j-2} \bar{b}_{1}, \varepsilon_{j-2} \bar{b}_{2}\right),\left(\varepsilon_{j-1} \bar{b}_{0}, \varepsilon_{j-1} \bar{b}_{1}\right) \in H$ and since $\bar{b}_{0} \in B, \bar{b}_{k} \in A$ we have $\left(0, \bar{b}_{0}\right),\left(\bar{b}_{k}, 0\right) \in H$. Adding gives $\left(E\left(b_{j-1}\right) \cdot F_{1}, E\left(b_{j}\right) \cdot F_{2}\right) \in H$. Thus $f \in N_{0}$.

Claim 3. $f(A)=\{0\}$.

For $a \in A, E(a)=(0, \ldots, 0)$ so $f(a)=0$.

From Claims 2 and 3, $f \in \operatorname{Ann}_{N_{0}}(A)$. Moreover $B \nsubseteq A_{k}$ (for if $B \subseteq A_{k}$ then $\left|A_{k+1}\right|=\left|A_{k}+B\right|=\left|A_{k}\right|$ and since $A_{k} \subseteq A_{k+1}$ then $A_{k}=A_{k+1}=A_{k+2}=$ $\cdots$, contradicting $n=A l(G)$ ) so for $b \in B \backslash A_{k}, f(b)=\bar{b}_{0} \neq 0$ hence we have the desired function.

In a sequence of lemmas we establish the converse. For convenience we say $H=G / A \stackrel{\times}{\sim}_{\sigma} G / B$ has property $\Sigma$ if $\exists n, n=A l(G)$ and $\forall k, 0 \leq k<n$, $A \cap B_{k}=\{0\}$.

Lemma III.6. Let $H$ satisfy property $\Sigma$. Then for each $i, 0 \leq i \leq n,\left|A_{i}\right|=$ $\left|B_{i}\right|=|A|^{i+1}$. Therefore $|G|=|A|^{n+1}$ and $B l(G)=n$.

Proof. We know $\left|A_{j}\right|=\left|A_{j-1}+B\right|$ for all $j, 1 \leq j \leq n$. Since $A_{j-1} \cap B=\{0\}$, $\left|A_{j}\right|=\left|A_{j-1}\right||B|$. From $\left|A_{1}\right|=|A||B|=|A|^{2}$ we get $\left|A_{j}\right|=|A|^{j+1}$, hence $|G|=\left|A_{n}\right|=|A|^{n+1}$. From Lemma II.3, $B \cap A_{k}=\{0\} \quad \forall k, 0 \leq k<n$ so in a symmetric manner we get $\left|B_{n}\right|=|A|^{n+1}$, hence $B_{n}=G$. But this means $B l(G)=n$.

Let $\psi: N_{0} \rightarrow M_{0}(A)$ be the restriction map, $\psi(f)=f / A$. It is clear that $\psi$ is a near ring homomorphism. When $H$ satisfies property $\Sigma$ we show $\psi$ is an isomorphism. Since $M_{0}(A)$ is simple we will have the converse of Theorem III.5. We first show $\psi$ is one to one. 
Lemma III.7. Let $H$ satisfy property $\Sigma$.

(i) $\forall j, 0 \leq j \leq n, A_{j} \cap B_{n-j-1}=\{0\}$.

(ii) $\left(A_{1} \cap B_{n-1}\right)+\left(A_{2} \cap B_{n-2}\right)+\cdots+\left(A_{n-1} \cap B_{1}\right)$ is a direct sum.

(iii) $A_{n-1} \cap B_{n-1}=\sum_{i=1}^{n-1} \oplus\left(A_{i} \cap B_{n-i}\right)$.

(iv) $G=A \oplus B \oplus\left(A_{n-1} \cap B_{n-1}\right)$.

Proof. (i) Let $x \in A_{j} \cap B_{n-j-1}$. Then $\exists a_{j-1}, \ldots, a_{0}$ such that $\left(x, a_{j-1}\right)$, $\left(a_{j-1}, a_{j-2}\right), \ldots,\left(a_{1}, a_{0}\right) \in H$ where $a_{i} \in A_{i} \cap B_{n-i-1}$. Thus $a_{0}=0$ and from this $a_{1}=a_{2}=\cdots=a_{j-1}=x=0$.

(ii) It suffices to show $\left(A_{1} \cap B_{n-1}\right)+\cdots+\left(A_{i-1} \cap B_{n-(i-1)}\right) \cap\left(A_{i} \cap B_{n-i}\right)=\{0\}$, for all $i, 2 \leq i \leq n-1$. But this is immediate since $x \in\left(A_{1} \cap B_{n-1}\right)+\cdots+$ $\left(A_{i-1} \cap B_{n-(i-1)}\right) \cap\left(A_{i} \cap B_{n-i}\right)$ implies $x \in A_{i-1} \cap B_{n-i}=\{0\}$ by part (i).

(iii) Consider $A_{i} \cap B_{n-i}$ for any $i, 1 \leq i \leq n-1$. From part (i) $A_{i-1} \cap\left(A_{i} \cap\right.$ $\left.B_{n-i}\right)=\{0\}$ so $A_{i-1} \oplus\left(A_{i} \cap B_{n-i}\right)$ is a subgroup of $A_{i}$ with order $|A|^{i}\left|A_{i} \cap B_{n-i}\right|$. By Lemma III.4, $\left|A_{i} \cap B_{n-i}\right|=|A|$ and therefore $\left|A_{i-1} \oplus A_{i} \cap B_{n-i}\right|=|A|^{i}|A|=$ $|A|^{i+1}$. This in turn implies $\left|\sum_{i=1}^{n-1} \oplus\left(A_{i} \cap B_{n-i}\right)\right|=|A|^{n-1}$. On the other hand, from $|A|^{n+1}=|G| \geq\left|A_{n-1}+B_{n-1}\right|=|A|^{2 n} /\left|A_{n-1} \cap B_{n-1}\right|$ we get $\left|A_{n-1} \cap B_{n-1}\right| \geq$ $|A|^{n-1}$ and since $A \cap\left(A_{n-1} \cap B_{n-1}\right)=\{0\},\left|A \oplus\left(A_{n-1} \cap B_{n-1}\right)\right|=|A| \mid A_{n-1} \cap$ $\left.B_{n-1}|\leq| A\right|^{n}$ or $\left|A_{n-1} \cap B_{n-1}\right|=|A|^{n-1}$. Since $\sum_{i=1}^{n-1} \oplus\left(A_{i} \cap B_{n-i}\right) \subseteq A_{n-1} \cap B_{n-1}$ the result follows.

(iv) It is clear that $A \oplus B \cap\left(A_{n-1} \cap B_{n-1}\right)=\{0\}$. Hence $A \oplus B \oplus\left(A_{n-1} \cap B_{n-1}\right)$ is a subgroup of $G$ of order $|A||A||A|^{n-1}=|A|^{n+1}$, i.e. $G=A \oplus B \oplus A_{n-1} \cap B_{n-1}$.

Corollary III.8. Let $H$ satisfy property $\Sigma$ and let $w \in G$. Then $w=a+b+c$, $a \in A, b \in B, c \in A_{n-1} \cap B_{n-1}$ and $\forall f \in N_{0}, f(w)=f(a+b+c)=$ $\hat{a}+\hat{b}+f(c), \hat{a} \in A, \hat{b} \in B$.

Proof. From part (iv) of the above lemma, $w=a+b+c, a \in A, b \in B$, $c \in A_{n-1} \cap B_{n-1}$. From Lemma II.7, $f(a+b+c)=f(b+c+a)=f(b+c)+\hat{a}$, $\hat{a} \in A$ and $f(b+c)=f(c+b)=f(c)+\hat{b}, \hat{b} \in B$.

Lemma III.9. Let $H$ satisfy property $\Sigma$ and let $f \in \operatorname{Ann}_{N_{0}}(A)$.

(i) $f\left(A_{j}\right) \subseteq A_{j-1} \quad \forall_{j}, \quad 1 \leq j \leq n$.

(ii) $f(B)=\{0\}$.

(iii) $f\left(B_{j}\right) \subseteq B_{j-1}, \forall_{j}, \quad 1 \leq j \leq n$.

(iv) $f(A \oplus B)=\{0\}$.

(v) $f(G) \subseteq A_{n-1} \cap B_{n-1}$.

Proof. (i) Let $a_{1} \in A_{1}$. Thus $\exists a_{0} \in A$ such that $\left(a_{1}, a_{0}\right) \in H$. But then $\left(f\left(a_{1}\right), f\left(a_{0}\right)\right)=\left(f\left(a_{1}\right), 0\right) \in H$ implies $f\left(a_{1}\right) \in A=A_{0}$. Continuing, let $a_{j} \in$ $A_{j}$ and suppose $f\left(A_{j-1}\right) \subseteq A_{j-2}$. Then for some $a_{j-1} \in A_{j-1},\left(a_{j}, a_{j-1}\right) \in H$ so $\left(f\left(a_{j}\right), f\left(a_{j-1}\right)\right) \in H$. Since $f\left(a_{j-1}\right) \in A_{j-2}, f\left(a_{j}\right) \in A_{j-1}$.

(ii) From part (i), $f(G)=f\left(A_{n}\right) \subseteq A_{n-1}$ and so $f(B) \subseteq A_{n-1}$. But from Lemma II.1, $f(B) \subseteq B$ so we have $f(B) \subseteq A_{n-1} \cap B=\{0\}$. 
(iii) Follows from (ii) using the same arguments as in (i).

(iv) Follows from (ii) and Corollary II.8.

(v) From (i) and (iii), $f(G)=f\left(A_{n}\right) \subseteq A_{n-1}$ and $f(G)=f\left(B_{n}\right) \subseteq B_{n-1}$.

Lemma III.10. If $f \in N_{0}$ and $f(A)=\{0\}$ then $f(G)=\{0\}$.

Proof. Let $f \in N_{0}$ such that $f \in \operatorname{Ker} \psi$, i.e. $f(A)=\{0\}$. For $x \in G$, from Corollary III.8, $x=a+b+c, a \in A, b \in B, c \in A_{n-1} \cap B_{n-1}$ and $f(x)=$ $\hat{a}+\hat{b}+f(c), \hat{a} \in A, \hat{b} \in B$. From the above lemma, $f(x) \in A_{n-1} \cap B_{n-1}$. Thus if $f(c)=0$ then $f(x) \in\left(A_{n-1} \cap B_{n-1}\right) \cap A \oplus B=\{0\}$ from Lemma III.7, (iv). Therefore $f(A)=\{0\}$ implies $f$ is the zero map which means $\operatorname{Ker} \psi=\{0\}$. To complete the proof we show $f\left(A_{n-1} \cap B_{n-1}\right)=\{0\}$.

Let $w \in A_{n-1} \cap B_{n-1}, w=x_{1}+x_{2}+\cdots+x_{n-1}$ where $x_{i} \in A_{i} \cap B_{n-i}$, $i=1,2, \ldots, n-1$. If $w=0$ we are finished so we take $w \neq 0$. Let $l$ be the largest integer such that $x_{l} \neq 0$. If $l=1, w=x_{1}$ and $f(w) \in$ $f\left(A_{1} \cap B_{n-1}\right) \subseteq A \cap B_{n-2}=\{0\}$. If $l=2, w=x_{1}+x_{2}$. Since $x_{1} \in A_{1} \cap B_{n-1}$, $\exists a_{0} \in A \cap B_{n}$ such that $\left(x_{1}, a_{0}\right) \in H$. Also, since $x_{2} \in A_{2} \cap B_{n-2}, \exists a_{1} \in$ $A_{1} \cap B_{n-1}$ with $\left(x_{2}, a_{1}\right) \in H$. Therefore $\left(x_{1}+x_{2}, a_{0}+a_{1}\right) \in H$ and thus $\left(f\left(x_{1}+x_{2}\right), f\left(a_{0}+a_{1}\right)\right) \in H$. However, from Lemma II.7, $f\left(a_{0}+a_{1}\right)=\hat{a}_{0}+$ $f\left(a_{1}\right), \hat{a}_{0} \in A$ and by the previous case $f\left(a_{1}\right)=0$. Now $\left(f\left(x_{1}+x_{2}\right), \hat{a}_{0}\right) \in H$ implies $f\left(x_{1}+x_{2}\right) \in A_{1}$. But $x_{1}, x_{2} \in B_{n-1}$ imply $f\left(x_{1}+x_{2}\right) \in B_{n-2}$. Thus $f\left(x_{1}+x_{2}\right) \in A_{1} \cap B_{n-2}=\{0\}$ from Lemma III.7, (i). Assume the results for all elements of the form $x_{1}+\cdots+x_{l-1}$ and let $u=y_{1}+\cdots+y_{l}$. As above, $\exists a_{i} \in A_{i} \cap B_{n-i}$ with $\left(y_{i+1}, a_{i}\right) \in H, 0 \leq i \leq l-1$ and we obtain $\left(f(u), f\left(a_{0}+\cdots+a_{l-1}\right)\right)=\left(f(u), \hat{a}_{0}+f\left(a_{1}+\cdots+a_{l-1}\right)\right)=\left(f(u), \hat{a}_{0}\right)$ by the induction hypothesis. Hence $f(u) \in A_{1}$ and $u \in B_{n-1}$ implies $f(u) \in B_{n-2}$, so we have $f(u)=0$. The result follows by induction.

We now have that $\psi: N_{0} \rightarrow M_{0}(A)$ defined by $\psi(f)=f / A$ is a monomorphism when $H$ satisfies property $\Sigma$. It remains to show that every function $f$ on $A$ with $f(0)=0$ can be extended to a function in $N_{0}$.

From Lemma III.4 we have isomorphisms $\sigma_{j}$,

$$
\begin{aligned}
B= & A_{n} \cap B_{0} \stackrel{\sigma_{n}}{\rightarrow} A_{n-1} \cap B_{1} \stackrel{\sigma_{n-1}}{\rightarrow} \cdots \rightarrow A_{j} \cap B_{n-j} \stackrel{\sigma_{j}}{\rightarrow} A_{j-1} \cap B_{n-(j-1)} \rightarrow \cdots \rightarrow \\
& A_{1} \cap B_{n-1} \stackrel{\sigma_{1}}{\rightarrow} A_{0} \cap B_{n}=A .
\end{aligned}
$$

Let $f_{0} \in M_{0}(A)$. We extend $f_{0}$ to a function $f \in N_{0}$. First let $f=f_{0}$ on $A=A_{0} \cap B_{n}$. We extend to $A_{1} \cap B_{n-1}$. Let $x \in A_{1} \cap B_{n-1}$. From the definition of $\sigma_{1},\left(x, \sigma_{1}(x)\right) \in H$ and $\sigma_{1}(x) \in A_{0}$. But then $f \sigma_{1}(x) \in A$. We define $f(x)$ to be the unique element in $A_{1} \cap B_{n-1}$ such that $\sigma_{1} f(x)=f \sigma_{1}(x)$. Assume now we have defined $f$ on $A_{j-1} \cap B_{n-(j-1)}$ and let $x \in A_{j} \cap B_{n-j}$. Then $\left(x, \sigma_{j}(x)\right) \in H, \sigma_{j}(x) \in A_{j-1} \cap B_{n-(j-1)}$ and $f \sigma_{j}(x) \in A_{j-1} \cap B_{n-(j-1)}$. Define $f(x)$ to be the unique element in $A_{j} \cap B_{n-j}$ such that $\sigma_{j} f(x)=f \sigma_{j}(x)$. 
Therefore $\forall j, 0 \leq j \leq n$ we have $f$ defined on $A_{j} \cap B_{n-j}$ and $f \sigma_{j}(x)=\sigma_{j} f(x)$ $\forall x \in A_{j} \cap B_{n-j}$. Recall from Lemma III.7 (iv), $G=\left(A \cap B_{n}\right) \oplus\left(A_{1} \cap B_{n-1}\right) \oplus$ $\cdots \oplus\left(A_{n-1} \cap B_{1}\right) \oplus\left(A_{n} \cap B\right)$. We extend $f$ linearly to $G$, i.e. for $x \in G$, $x=\sum_{i=0}^{n} x_{i}$ where $x_{i} \in A_{i} \cap B_{n-i}$ define $f(x)=\sum_{i=0}^{n} f\left(x_{i}\right)$.

We next show that $f \in N_{0}$. Let $(u, v) \in H, u=\sum_{i=0}^{n} u_{i}, u_{i} \in A_{i} \cap B_{n-i}$ and $v=\sum_{i=0}^{n} v_{i}, v_{i} \in A_{i} \cap B_{n-i}$. For $i=1,2, \ldots, n,\left(u_{i}, \sigma_{i}\left(u_{i}\right)\right) \in H$ and since $\left(u_{0}, 0\right) \in H$, we have $\left(u, \sigma_{1}\left(u_{1}\right)+\cdots+\sigma_{n}\left(u_{n}\right)\right) \in H$. From $(u, v) \in H$ and $\left(0, v_{n}\right) \in H$ we get $\left(u, v_{0}+v_{1}+\cdots+v_{n-1}\right) \in H$. Thus $\left(0, \sigma_{1}\left(u_{1}\right)-v_{0}+\right.$ $\left.\sigma_{2}\left(u_{2}\right)-v_{1}+\cdots+\sigma_{n}\left(u_{n}\right)^{-}-v_{n-1}\right)$ is in $H$ so $\sigma_{1}\left(u_{1}\right)-v_{0}+\cdots+\sigma_{n}\left(u_{n}\right)-v_{n-1} \in B$. But also $\sigma_{1}\left(u_{1}\right)-v_{0}+\cdots+\sigma_{n}\left(u_{n}\right)-v_{n-1} \in A_{0}+\cdots+A_{n-1}=A_{n-1}$, hence $\sigma_{1}\left(u_{1}\right)-$ $v_{0}+\cdots+\sigma_{n}\left(u_{n}\right)-v_{n-1}=0$ which in turn gives $v=\sigma_{1}\left(u_{1}\right)+\cdots+\sigma_{n}\left(u_{n}\right)+v_{n}$. Therefore $f(v)=f \sigma_{1}\left(u_{1}\right)+\cdots+f \sigma_{n}\left(u_{n}\right)+f\left(v_{n}\right)=\sigma_{1} f\left(u_{1}\right)+\cdots+\sigma_{n} f\left(u_{n}\right)+f\left(v_{n}\right)$ and $f(u)=\sum_{i=0}^{n} f\left(u_{i}\right)$. But, $\left(f\left(u_{0}\right), 0\right) \in H,\left(f\left(u_{i}\right), \sigma_{i} f\left(u_{i}\right)\right) \in H$ for $i=1,2, \ldots, n$ and $\left(0, f\left(v_{n}\right)\right) \in H$ together imply that $(f(u), f(v)) \in H$, consequently $f \in N_{0}$. Combining this with the previous lemma gives the following.

Theorem III.11. If $\exists n, n \in A l(G)$ and $\forall k<n \quad A \cap B_{k}=\{0\}$ then $N_{0}$ is simple.

Combining Theorems III.11 and III.5 we have our major result.

Theorem III.12. Let $H=G / A \stackrel{\times}{\sim} \sigma G / B, A \neq\{0\} \neq B, A \cap B=\{0\}$. $M_{0}(G, 2, H)$ is simple $\Leftrightarrow \exists n, n=A l(G)$ and $\forall k, 0 \leq k<n, A \cap B_{k}=\{0\}$.

We conclude this section and the paper by showing that for the near-ring $M_{0}(G, 2, H)$ the concepts of simplicity and 2-semisimplicity coincide. This is identical with the situation for centralizer near-rings $\mathscr{C}(\mathscr{A} ; G)$ when $\mathscr{A}$ is a cyclic group of automorphisms.

Theorem III.13. Let $H=G / A \stackrel{\times}{\sim} G / B, A \cap B=\{0\}, N_{0}$ is simple $\Leftrightarrow N_{0}$ is 2-semisimple.

Proof. If $A=\{0\}$ then as in Theorem II.6, $N_{0}$ is the centralizer near-ring $\mathscr{C}(\mathscr{A} ; G)$ where $\mathscr{A}=\langle\sigma\rangle$. Consequently this case follows from [2].

Now take $A \neq\{0\}$ and suppose $N_{0}$ is not simple. If $\forall n, A_{n} \neq G$ we let $l$ be the least index such that $A_{l}=A_{l+1}$. Let $I=\left\{f \in N_{0} \mid f\left(A_{l}\right)=\{0\}\right.$ and $\left.f\left(G \backslash A_{l}\right) \subseteq A_{l}\right\}$. Then $I$ is a nilpotent $N_{0}$-subgroup. Suppose $\exists a_{l} \in A_{l}$, $a_{l} \neq 0$ such that $\left(a_{l}, a_{l}\right) \in H$. Define $f: G \rightarrow G$ by $f(x)=0$ if $x \in A_{l}$ and $f(x)=a_{l}$ if $x \notin A_{l}$. Since $A_{l}=A_{l+1}=\cdots, B \subseteq A_{l}$, consequently, for $(x, y) \in H, x \in A_{l} \Leftrightarrow y \in A_{l}$. If $x \notin A_{l},(f(x), f(y))=\left(a_{l}, a_{l}\right) \in H$ while if $x \in A_{l},(f(x), f(y))=(0,0) \in H$. Thus $f \in I$ and $I \neq\{0\}$. It remains to show that such an $a_{l}$ exists. Since $l$ is least with $A_{l}=A_{l+1}=\cdots$, $\exists b \in B, b \neq 0, b \in A_{l} \backslash A_{l-1}$. Further, since $b \in A_{l}, \sigma(b+A)=a_{l-1}+B$, $a_{l-1} \in A_{l-1}$. But then $\sigma\left(a_{l-1}+A\right)=a_{l-2}+B, a_{l-2} \in A_{l-2}$ and continuing we obtain $\sigma\left(a_{l-2}+A\right)=a_{l-3}+B, \ldots, \sigma\left(a_{1}+A\right)=a_{0}+B, \sigma\left(a_{0}+A\right)=\sigma(A)=B$. Adding gives $\sigma\left(b+a_{l-1}+a_{l-2}+\cdots+a_{1}+A\right)=a_{l-1}+a_{l-2}+\cdots+a_{0}+B$. Now let 
$a_{l}=b+a_{l-1}+a_{l-2}+\cdots+a_{1}+a_{0}$. Then $a_{l} \in\left(b+a_{l-1}+\cdots+a_{1}+A\right) \cap$ $\left(a_{l-1}+\cdots+a_{0}+B\right)$ so $\left(a_{l}, a_{l}\right) \in H$ and $a_{l} \neq 0$ since $b \notin A_{l-1}$ while $a_{l-1}+a_{l-2}+\cdots+a_{0} \in A_{l-1}$.

If $\exists n, n=A l(G)$ and $N_{0}$ is not simple, then as in the proof of Theorem III.5 we get that $\operatorname{Ann}_{N_{0}}(A)$ is a nonzero $N_{0}$-subgroup of $N_{0}$. We note that if $f \in \operatorname{Ann}_{N_{0}}(N)$ then $f\left(A_{1}\right) \subseteq A_{0}, f\left(A_{2}\right) \subseteq A_{1}, \ldots$, and $f(G)=f\left(A_{n}\right) \subseteq$ $A_{n-1}$. From this we have the product of any $n$ elements in $\operatorname{Ann}_{N_{0}}(A)$ is 0 , so $\operatorname{Ann}_{N_{0}}(A)$ is nilpotent and again $N_{0}$ is not 2-semisimple.

\section{REFERENCES}

1. P. Fuchs and C. J. Maxson, Near-fields associated with invariant linear k-relations, Proc. Amer. Math. Soc. 103 (1988), 729-736.

2. C. J. Maxson, and K. C. Smith, The centralizer of a group automorphism, J. Algebra 54 (1978), 27-41.

3. J. D. P. Meldrum, Near-rings and their links with groups, Research Notes in Math. 134, Pitman Publ. Co., London, 1986.

4. G. F. Pilz, Near-rings, 2nd Ed., North Holland, Amsterdam, 1983.

5. R. Remak, Über die Darstellung der endlichen Gruppen als Untergruppen direkter Produkte, J. Reine Angew. Math. 163 (1930), 1-44.

6. H. Wielandt, How to single out function near-rings, Oberwolfach Abstracts, 1972.

Department of Mathematics, Texas A\&M University, College Station, Texas 77843 